\title{
Progress in Optical Parametric Oscillators
}

\author{
M. Ebrahim-Zadeh ${ }^{1,2}$ \\ ${ }^{1}$ ICFO-Institut de Ciencies Fotoniques, Mediterranean Technology Park, 08860 Castelldefels, Barcelona, Spain \\ ${ }^{2}$ Institucio Catalana de Recerca i Estudis Avancats (ICREA), Passeig Lluis Companys 23, Barcelona 08010, Spain
}

Optical parametric oscillators are uniquely versatile sources of tunable coherent radiation in spectral regions inaccessible to conventional lasers [1]. The advent of novel nonlinear optical materials over the past two decades, combined with progress in laser pump sources, has had an unprecedented impact on OPOs, enabling advancement of this technology to new spectral and temporal domains. The rapid developments have led to the realization of a new generation of OPO sources capable of providing widely tunable radiation with unparalleled performance capabilities with regard to wavelength coverage, output power and efficiency, spatial, spectral and temporal coherence across vast spectral regions. Harnessing the superior linear and nonlinear optical properties of new birefringent and quasi-phase-matched materials, together with the application of innovative design concepts, combined with novel solid-state and fiber pump lasers, OPOs have been transformed from proof-of-concept laboratory prototypes to truly viable sources of tunable coherent radiation from $\sim 250 \mathrm{~nm}$ in the UV up to $\sim 12 \mu \mathrm{m}$ in the deep-IR, and in all temporal domains from continuous-wave to few-cycle femtosecond time-scales [1-3]. The latest examples of progress in OPOs include broadband phase-locked pulse generation in picosecond timescale [4], see Fig. 1, and widely tunable soliton generation in the femtosecond domain [5], shown in Fig. 2.
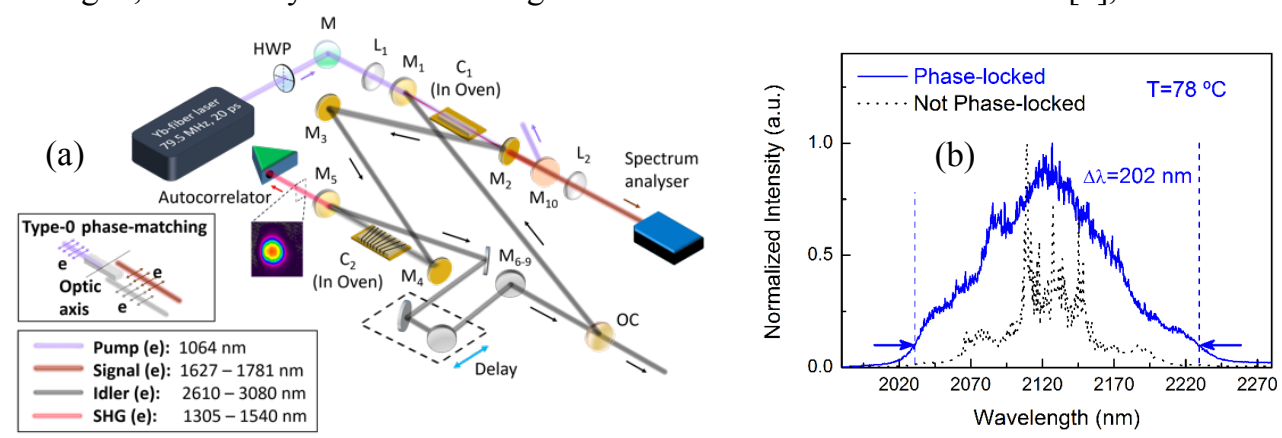

Fig. 1 (a) Yb-fiber-pumped phase-locked degenerate picosecond OPO based on MgO:PPLN for broadband generation in the near-infrared. (b) OPO output spectrum in the absence of phase-locking and in degenerate phase-locked state [4]
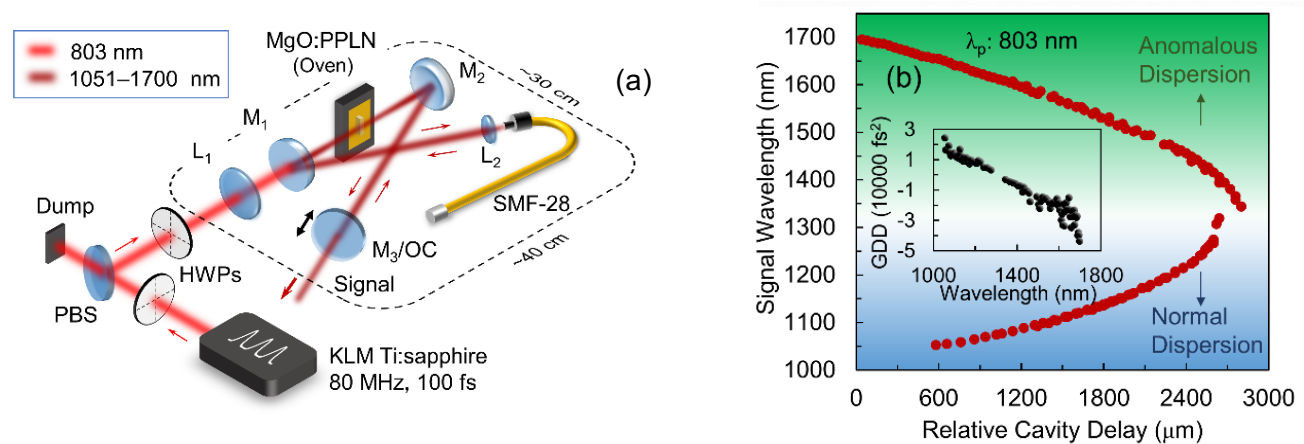

Fig. 2 (a) Ti:sapphire pumped soliton fibre-feedback OPO. (b) Cavity delay tuning of the OPO; inset: calculated net cavity GDD across the tuning range [5].

This lecture will provide an overview of parametric sources, from the basic design concepts to advanced systems, also highlighting the latest progress at the frontiers of OPO technology from the ultraviolet to the deep-infrared and from the continuous-wave to the ultrafast few-cycle operating time-scales. The lecture will include a description of nonlinear materials, innovative device architectures, applications of OPO sources in spectroscopy, imaging, environmental sensing, life sciences and biomedicine, and industrial development of OPO technology.

\section{References}

[1] M. Ebrahim-Zadeh and M. H. Dunn, "Optical parametric oscillators," in Handbook of Optics IV, 2nd ed., Optical Society of America, McGraw-Hill, New York, Ch. 22, pp. 22.01-22.72 (2001).

[2] M. Ebrahim-Zadeh, "Mid-infrared ultrafast and continuous-wave optical parametric oscillators," in Solid-State Mid-Infrared Laser Sources, I. T. Sorokina and K. L. Vodopyanov eds., Springer-Verlag, Berlin, Topics Appl. Phys. 89, 179-219 (2003).

[3] M. Ebrahim-Zadeh, "Continuous-wave optical parametric oscillators", in Handbook of Optics IV, 3rd ed., Optical Society of America, McGraw-Hill, New York, Ch. 17, pp. 17.1-17.33 (2010).

[4] B. Nandy, S. Chaitanya Kumar, M. Ebrahim-Zadeh, "Phase-locked picosecond optical parametric oscillator”, Opt. Lett. 45, 3981 (2020).

[5] C. F. O'Donnell, S. Chaitanya Kumar, T. Paoletta, M. Ebrahim-Zadeh, "Widely tunable femtosecond soliton generation in a fiber-

feedback optical parametric oscillator", Optica 7, 426 (2020). 